\title{
Avaliação das ações de educação na saúde bucal de adolescentes dentro da Estratégia de Saúde da Família
}

\author{
Assessment of educational actions on the oral health \\ of adolescents within the Family H ealth Strategy
}

\author{
Ana Paula Silveira Turrioni ${ }^{1}$ \\ Fernanda Gonçalves Duvra Salomão ${ }^{2}$ \\ José Fernando Casquel M onti ${ }^{3}$ \\ Fabiana de Lima Vazquez ${ }^{2}$ \\ Karine Laura Cortellazzi ${ }^{2}$ \\ Antonio Carlos Pereira ${ }^{2}$
}

\footnotetext{
${ }^{1}$ Residência

Multiprofissional em Saúde da Família e Comunidade, UniversidadeFederal deSão Carlos. Rodovia Washington Luís, km 235. 13565-905 São Carlos São Paulo.

apereira@fop.unicamp.br ${ }^{2}$ Departamento de Odontologia Social, Faculdadede Odontologia dePiracicaba, U niversidade Estadual deCampinas. ${ }^{3}$ Divisão de Dermatologia, Instituto Lauro deSouza Lima, Coordenadoria de ControledeDoenças.
}

A bstract 0 bjective: to evaluate the actions of education on the oral health of adolescents within the scope of the Family Health Strategy. M ethodology: A questionnaire was applied and a clinical exam conducted evaluating biofilm and gingival inflammation in 80 adolescents before and after interventions. A qualitative study of the brushing technique was al so performed on 15 adolescents in the sample. Results: therewas an improvement in oral hygiene of adolescents who participated in actions at school and an increase in the number of adolescents who had healthy gums or only mild inflammation in the second test in school groups and home visits. With respect to the consumption of candies, both the weekly group and the school group saw a decrease in candy consumption between the first and second tests. There was an increased frequency of brushing in the school group. Conclusion: considering the importance of educational activities observed in this study, the process of training and joint planning of activities among theschool staff and health professionals would appear to be important.

Key words $\mathrm{H}$ ealth education, O ral health, Adolescents
Resumo Objetivo: avaliar as ações de educação na saúde bucal deadolescentes dentro da Estraté gia de Saúde da Família. M etodologia: foi aplicado um questionário e realizado exame clínico avaliando biofilme e inflamação gengival em 80 adolescentes antes e depois das intervenções. U m estudo qualitativo da técnica de escovação também foi realizado em 15 adolescentes pertencentes à amostra. Resultados: observou-se melhora na higiene oral dos que participaram das ações na escola, aumento no número daqueles que apresentaram gengiva saudável ou inflamação leve no segundo exame nos grupos escola e visita domiciliar. Com relação ao consumo de doces, o grupo semanal e da escola apresentou diminuição entre 0 1ㅇe $2^{2}$ exames. H ouveum aumento na frequência de escovação no grupo escola. Conclusão: diante da importância das ações educativas verificadas neste estudo, parece nos importante o processo de capacitação e o planejamento conjunto das ações entre os profissionais da escola e os profissionais de saúde.

Palavras-chave Educação em saúde, Saúde bucal, Adolescentes 


\section{Introdução}

$\mathrm{Na}$ odontologia, o aparato tecnológico tem-se desenvolvido de forma uniforme no mundo globalizado, apresentando desenvolvimento técnico científico acentuado, porém não se tem observado resultados positivos na qualidade de vida relacionada à saúde bucal das pessoas ${ }^{1,2}$. No Brasil, apesar de alguns avanços nas últimas décadas, a assistência odontológica tem contribuído pouco para a melhoria das condições de saúde bucal da população, gerando um questionamento em relação à forma de atuação $0^{3,4}$.

0 cirurgião-dentista que trabal ha na Estraté gia de Saúde da Família (ESF) vê-se frequentemente diante de muitos desafios para o exercício de suas atividades. Na maioria das vezes, o profissional não está preparado para atividades de promoção e prevenção, realizando-as de maneira inadequada ou incompleta. Tais atividades são efetuadas, geralmente, com aplicação tópica de flúor, orientações individuais na hora da consulta epalestras com cerca de meia hora de duração, de maneira expositiva, sem a participação do usuário na construção de saberes que os capacitem nas questões relativas ao desenvolvimento de seu autocuidado. A população respondea estas ações, ávida pelo tratamento curativo, resistindo a cada palestra e a cada reunião ${ }^{3,5}$.

A inserção da equipe desaúde bucal na Estratégia de Saúde da Família deve fazer com que os cirurgiões-dentistas repensem suas práticas, valores e conhecimentos envolvidos na saúde. 0 processo saúde-doença relacionado com a cárie envolve outros fatores além do dente e do biofilme dental, tais como fatores de ordem social ${ }^{6}$. Uma estratégia promissora para enfrentar estes múltiplos problemas é a Promoção da Saúde, devendo haver uma articulação de saberes técnicos e populares, mobilização de recursos institucionais e comunitários, públicos e privados, associados a um conjunto de val ores como intersetorialidade, solidariedade, equidade, democracia, cidadania, desenvolvimento social, participação comunitária e parceria ${ }^{3}$. Outra importante estratégia é a Educação em Saúde, que tem o objetivo de oferecer instrumentos para fortalecer a autonomia dos usuários no controle do processo saúde-doença e na condução de seus hábitos. Devem-sedifundir elementos, respeitando a cultura local, que possam contribuir com o empoderamento dos sujeitos coletivos, tornando-os capazes de autogerirem seu processo de saúde-doença, sua vida, com vista à melhoria da sua qualidade de vida?
Com relação aos adolescentes, destaca-se a importância dese atuar nesta faixa devido às suas características singulares: rebeldia, busca deidentidade, instabilidadee desinteresse que setornam grandes barreiras para se conseguir efetividade das ações em saúde 8 . A adolescência étida como um período de risco para a cárie, em decorrência do precário controle de placa e da redução dos cuidados com a escovação dentária9. Além disto, no estudo de Ferrari et al. ${ }^{10}$, encontrou-se que 0 atendimento aos adolescentes naUnidade deSaúde da Família não é sistematizado por existirem outras prioridades. Eles não procuram o serviço e os profissionais se sentem despreparados para perceber o que ocorre e prestar o devido atendimento. No plano de metas Saúde Bucal Para Todos para o Ano 2010, preconiza-se que, aos 15 anos, não haja mais que um sextante com alguma necessidade de tratamento periodontal e a ausência de perda dental aos 18 anos devido à cárieeà doença periodontal ${ }^{11}$. A real idade do Brasil está muito distante das metas propostas, principalmente nos níveis populacionais de menor renda ${ }^{12}$. Baseado nestas considerações, o objetivo do presenteestudo foi analisar a interferência das ações de educação na saúde bucal deadolescentes dentro da Estratégia de Saúde da Família.

\section{M etodologia}

O projeto desta pesquisa foi aprovado pelo Comitê de Ética e Pesquisa em Seres Humanos da Universidade Federal de São Carlos (UFSCAR), e um termo deconsentimento livreeesclarecido foi adotado para autorização da participação voluntária e explicação sobre os termos da pesquisa. 0 estudo foi realizado na área de abrangência da Unidade deSaúdeda Família (USF) Antenor Garcia com os adolescentes que estudam nas $5 a s$ e 8as séries da escola municipal "Artur Natalino Derigge". O local do estudo é considerado pelo Índice Paulista deVulnerabilidade Social (IPVS), uma área de vulnerabilidade muito alta (grau 6).

Foram selecionados al eatoriamente, por meio das listas de presença, 80 adolescentes considerando a representatividade da amostra no total de 246 adolescentes do bairro cadastrados no SIAB (Sistema de Informação da Atenção Básica) representando $30,7 \%$ do total.

Os Escolares foram divididos em três grupos: (A) 55 adolescentes que participaram de atividadeseducativas desenvolvidas na escola; (B) 13 que tiveram ambientação em saúde bucal, através de orientação para as famílias, durante visitas do- 
miciliares mensais dos Agentes Comunitários de Saúde(ACS); (C) 12 que participaram deum grupo semanal de orientação para melhoria da qualidade de vida, tendo sido considerados os que participaram em mais de seis encontros.

Este estudo quali-quantitativo foi realizado em três etapas durante um período de quatro meses.

Na primeira etapa, foi aplicado um questionário pelo pesquisador, na forma de entrevista na escola com o objetivo de avaliar os hábitos de saúde bucal, sendo que as variáveis avaliadas no questionário foram: 1) consumo de doces; 2) consumo de salgados; 3) consumo de verduras e frutas; 4) consumo de cigarro; 5) consumo de bebida alcoólica; 6) obtenção de escova dental; 7) frequência de escovação; 8) uso do fio dental; 9) compartilhamento de escova dental. Após a aplicação do questionário, foi realizado um exameclínico no consultório odontológico da escola, utilizando sonda OM S e espel ho, para avaliar quantidade de placa (escores de 0 a 2 ) ${ }^{13}$ e condição gengival (escores de 0 a 3$)^{14} \mathrm{em}$ todos os participantes. É importante ressaltar que todos os adolescentes avaliados pertenciam à escola epassaram por exame clínico e entrevista.

$\mathrm{N}$ a segunda etapa foi real izada ações motivadoras para a criação de vínculo e aproximação dos adolescentes (pertencentes ao grupo C) com a USF, explorando os interesses e as habilidades de cada um. Posteriormente, foram realizadas ações educativas na escola, reuniões do grupo de adolescentes e visitas domiciliares dos ACS. 0 planejamento foi feito em conjunto com os professores e a coordenação. Tais ações incluíram:

- trabalho sobre o tema saúde bucal por cada professor em sua disciplina por dois meses; quatro encontros quinzenais (por sala), com roda de conversa entre a Dentista da USF e os adolescentes com os seguintes conteúdos:

1 ㅇe encontro: dinâmica para reflexão - "por que escovo e por que não escovo?". 0 objetivo foi sensibilizá-los em relação aos prejuízos, além da má higienebucal (convívio social, emprego, saúde geral).

$2^{\circ}$ encontro: apresentação com slides sobreo que são as bactérias e como ocorre a lesão de cáries, posteriormente foi apresentada a técnica de escovação.

3o encontro: evidenciação de placa e escovação supervisionada.

40 encontro: apresentação com slides reforçando a sensibilização, explicação de como ocorre a lesão cárie, a doença periodontal e as implicações destas doenças na vida e na saúde geral.
Ao final dos dois meses houve uma exposição do material, confeccionado pelos alunos durante o trabalho com os professores, para toda escola, inclusive de 1a a 4a série. Entre os materiais confeccionados relacionados com saúde bucal destacaram-se: desenhos relacionando bons e maus hábitos, jogos educativos (Educação Artística), experiências (Ciências), redações discutindo a importância de escovar os dentes (Português), elaboração de textos em inglês (Inglês) e cartazes apontando quantidade de Dentistas por região do país (Geografia). Também ocorreu a elaboração de teatro de fantoches (Português), teatro sobre a história da Odontologia (História) e análise estatística sobre a situação de risco na saúde bucal do bairro ( $M$ atemática). A Dentista disponibilizou materiais e algumas informações aos professores sempre que necessário.

Paral elamente às ações na escola, os ACS real izaram orientações de saúde bucal nas famílias dos adolescentes do grupo B. É importante ressaltar que os ACS participaram de uma capacitação com carga horária de 16 horas abordando assuntos como cárie, periodontite, câncer bucal e má oclusão, sendo criado um protocolo de visitas para orientação em saúde bucal, na tentativa de preparar devidamenteos agentes para abordar o assunto com as famílias.

No grupo $C$ foram realizados encontros abordando temas educativos gerais como: sexualidade, autoestima, planejamento do futuro, preconceito, entre outros. 0 objetivo principal do grupo foi a tentativa de aproximar os adolescentes da equipe de saúde, não sendo abordado somente temas relativos à saúde bucal.

$\mathrm{Na}$ terceira etapa, após um período de quatro meses, o questionário de hábitos de saúde bucal foi reaplicado, foi feito um $2^{\circ}$ exameclínico avaliando placa e condição gengival, e realizada uma $2^{a}$ entrevista subjetiva ( 15 adolescentes), para comparação e avaliação dos resultados obtidos nasintervenções.

Uma avaliação qualitativa da técnica de escovação também foi realizada em quinze adolescentes pertencentes à amostra estudada (cinco de cada grupo). Esta avaliação teve a finalidade de verificar seu grau de conhecimento, acerca da técnica de escovação. Sendo realizada logo após o exame clínico. Para tal avaliação, foi utilizado o método observacional, sendo pedido para que demonstrassem a forma como escovam seus dentes com o auxílio de um modelo de gesso. As falas e os gestos dos adolescentes foram registrados, por meio de gravação e anotações realizadas pelo pesquisador ${ }^{15}$. 
Para a análise dos dados de cada grupo foi realizado o teste de M c Nemar ao nível de significância de $5 \%$.

\section{Resultados}

A Tabela 1 indica os resultados do índice de higiene oral simplificado (IHO-S) em cada grupo. Pode-se perceber melhora na higiene oral dos adolescentes que participaram das ações na escola $(p=0,0021)$.

De acordo com a Tabela 2, observou-se um aumento no número de adolescentes que apresentaram gengiva saudável ou inflamação leve no segundo examenos grupos escola $(p \leq 0,0001)$ evisita domiciliar $(p=0,0313)$.

Com relação ao consumo de doces (Tabela 3) 0 grupo semanal $(p=0,0313)$ e da escola $(p=$ $0,0008)$ apresentou diminuição entre 010 e 2 은 exames.
A Tabela 4 mostra a frequência de escovação de cada grupo, onde houve um aumento no grupo escola $(p=0,0129)$.

Referente às informações fornecidas pelos adolescentes sobrea escovação, desmembraramse as falas e os gestos nas seguintes categorias: movimentos realizados com a escova de dente (horizontal, vertical, circular), faces dos dentes que eram higienizadas (vesti bular, lingual eoclusal), escovação da língua e se o adolescente apresentou uma sequência de regiões higienizadas. Em relação aos movimentos com a escova de dente, na demonstração realizada no primeiro momento da pesquisa, encontrou-se que seis adolescentes realizavam apenas um dos movimentos (sendo estes pertencentes aos grupos $\mathrm{B} \mathrm{eC}$ ) e nove realizavam a combinação de dois movimentos. 0 movimento mais utilizado foi o horizontal (15 adolescentes) eo menosutilizado foi o vertical (2 adolescentes). $\mathrm{Na}$ segunda entrevista, dois adolescentes realizaram apenas um movimento (per-

Tabela 1. Avaliação da condição de higiene bucal feita através do Índice de higiene oral simplificado (IHO-S) nos adolescentes dos três diferentes grupos de intervenção (escola, visita domiciliar e grupo) em dois momentos distintos ( 1 e e 2 oxame clínico).

\begin{tabular}{|c|c|c|c|c|}
\hline & Exame clínico & $\begin{array}{c}\text { Escola }(A)^{*} \\
(n=55) \\
n(\%)\end{array}$ & $\begin{array}{l}\text { VD }(B)^{*} \\
(n=13) \\
n(\%)\end{array}$ & $\begin{array}{c}\text { Grupo semanal }(C)^{*} \\
(n=12) \\
n(\%)\end{array}$ \\
\hline \multirow{7}{*}{ IHO-S } & Boa higiene & & & \\
\hline & 10. exame & $18(32,7)$ & $5(38,5)$ & $2(16,6)$ \\
\hline & 2‥ exame & $35(63,6)$ & $10(76,9)$ & $5(41,6)$ \\
\hline & Higiene razoável ou deficiente & & & \\
\hline & 1‥ exame & $37(67,3)$ & $8(61,5)$ & $10(83,3)$ \\
\hline & 2ㅇ. exame & $20(36,3)$ & $3(23,1)$ & $7(58,3)$ \\
\hline & $p$-value & $p=0,0021$ & $p=0,0625$ & $p=0,25$ \\
\hline
\end{tabular}

${ }^{*}$ Teste Mc Nemar $(p<0,05)$

Tabela 2. Avaliação feita através do Índice Gengival nos adolescentes dos três diferentes grupos de intervenção (escola, visita domiciliar e grupo) em dois momentos distintos ( 1 ํ e 2 ํ exame clínico).

\begin{tabular}{|c|c|c|c|c|}
\hline & Exame clínico & $\begin{array}{c}\text { Escola }(A)^{*} \\
\qquad \begin{array}{c}(n=55) \\
n(\%)\end{array}\end{array}$ & $\begin{array}{l}\text { VD }(B)^{*} \\
(n=13) \\
n(\%)\end{array}$ & $\begin{array}{c}\text { Grupo semanal }(C)^{*} \\
(n=12) \\
n(\%)\end{array}$ \\
\hline $\begin{array}{l}\text { Índice } \\
\text { Gengival }\end{array}$ & $\begin{array}{l}\text { Gengiva saudável e inflamação leve } \\
\text { 10. exame } \\
\text { 20. exame } \\
\text { Inflamação moderada e inflamação grave } \\
\text { 10. exame } \\
\text { 2o. exame } \\
\text { p-value }\end{array}$ & $\begin{array}{c}21(38,1) \\
41(74,6) \\
34(61,8) \\
14(25,4) \\
p \leq 0,0001\end{array}$ & $\begin{array}{c}4(30,8) \\
10(76,9) \\
9(69,2) \\
3(23,1) \\
p=0,0313\end{array}$ & $\begin{array}{l}6(50,0) \\
9(75,0) \\
6(50,0) \\
3(25,0) \\
p=0,25\end{array}$ \\
\hline
\end{tabular}

*Teste M c Nemar $(p<0,05)$ 
Tabela 3. Avaliação do consumo de doces feita através da aplicação do questionário nos adolescentes dos três diferentes grupos de intervenção (escola, visita domiciliar e grupo) em dois momentos distintos ( 10 e e $2^{\circ}$ exame).

\begin{tabular}{|c|c|c|c|c|}
\hline & Resultados & $\begin{array}{c}\text { Escola (A)* } \\
(n=55) \\
n(\%)\end{array}$ & $\begin{array}{l}\text { VD }(B)^{*} \\
(n=13) \\
n(\%)\end{array}$ & $\begin{array}{c}\text { Grupo semanal }(\mathrm{C}) \\
(\mathrm{n}=12) \\
\mathrm{n}(\%)\end{array}$ \\
\hline \multirow{6}{*}{ Consumo de doces } & Sempre & & & \\
\hline & 10. exame & $32(58,2)$ & $7(53,8)$ & $10(83,3)$ \\
\hline & 20. exame & $16(29,1)$ & $4(30,8)$ & $4(33,3)$ \\
\hline & 1o. exame & $23(41,8)$ & $6(46,2)$ & $2(167)$ \\
\hline & 20. exame & $39(70,9)$ & $9(69,2)$ & $8(66,7)$ \\
\hline & $p$-value & $p=0,0008$ & $p=0,3750$ & $p=0,0313$ \\
\hline
\end{tabular}

${ }^{*}$ Teste Mc Nemar $(p<0,05)$

Tabela 4. Avaliação da frequência de escovação feita através da aplicação do questionário nos adolescentes dos três diferentes grupos de intervenção (escola, visita domiciliar e grupo) em dois momentos distintos ( 1 e e $2^{\circ}$ exame).

\begin{tabular}{|c|c|c|c|c|}
\hline & Resultados & $\begin{array}{c}\text { Escola }(A)^{*} \\
(n=55) \\
n(\%)\end{array}$ & $\begin{array}{c}\text { VD }(B)^{*} \\
(n=13) \\
n(\%)\end{array}$ & $\begin{array}{c}\text { Grupo semanal }(\mathrm{C}) \\
(\mathrm{n}=12) \\
\mathrm{n}(\%)\end{array}$ \\
\hline \multirow{7}{*}{ Frequência de escovação } & Uma ou nenhuma & & & \\
\hline & 10. exame & $21(38,2)$ & $3(23,1)$ & $1(8,3)$ \\
\hline & 2ㅇ. exame & $9(16,3)$ & $2(15,38)$ & $1(8,3)$ \\
\hline & Duas ou mais & & & \\
\hline & 1‥ exame & $34(61,8)$ & $10(76,9)$ & $11(91,6)$ \\
\hline & 2ㅇ. exame & $46(83,6)$ & $11(84,6)$ & $11(91,6)$ \\
\hline & $p$-value & $p=0,0129$ & $p=1,000$ & $p=1,000$ \\
\hline
\end{tabular}

${ }^{*}$ Teste Mc Nemar $(p<0,05)$

tencentes ao grupo C) e treze realizaram a combinação de dois ou mais movimentos. 0 movimento horizontal continuou sendo o mais praticado. Houve aumento no número de adolescentes que realizaram o movimento vertical ( 7 adolescentes) e o movimento menos utilizado foi 0 circular (4 adolescentes). Pode-se perceber que os adolescentes passaram a realizar a combinação de movimentos o que é favorável na remoção da placa.

Com relação às faces dos dentes higienizadas, na primeira entrevista oito adolescentes higienizaram todas as faces esete não higienizaram a facelingual (adolescentes dos grupos A, B eC). $\mathrm{Na}$ segunda entrevista, doze adolescentes higienizaram todas as faces e três não higienizaram a facelingual (adolescentes do grupo A eC), apontando que houve aumento no número de adolescentes que higi enizaram todas as faces. Em relação ao relato de escovação da língua, no pri- meiro momento da pesquisa, oito adolescentes relataram sua higienização, sendo que no segundo momento, o número aumentou para doze adolescentes. Em relação à sequência de escovação, no primeiro momento cinco adolescentes possuíam uma sequência (adolescentes dos grupos $A$ e B) e na segunda entrevista o número aumentou para quinze. Ou seja, os adolescentes passaram a ter uma sequência no momento de escovação e percebe-se que a melhora na técnica de escovação foi evidente em todos os grupos.

\section{Discussão}

Vale ressaltar que este estudo não teve como objetivo unicamente medir o estado de saúde bucal dos adolescentes, como ocorrido em outros estudo ${ }^{16,17}$ e sim avaliar se as ações realizadas provocaram melhorias nas condições de saúde bu- 
cal, como realizado no estudo de Toassi e Petry ${ }^{18}$. A faixa etária foi escolhida representando adolescentes que pouco frequentam a unidade de saúde, por ser uma das divisões etárias do SIAB (Sistema de Informação da Atenção Básica) epor existirem poucas ações realizadas pela USF com esse grupo de pessoas.

A amostra examinada caracterizou-se, no primeiro instante, por indivíduos que apresentavam grande quantidade de biofilme, inflamação gengival e hábitos ruins em relação a saúde bucal. A maioria encontrava-secom higienedeficiente e com inflamação de moderada a grave. Estes resultados são semelhantes aos encontrados no estudo de Santos et al. ${ }^{16}$, que pesquisou as condições de saúde bucal de 1383 adolescentes no município deFeira de Santana, os quais apresentaram $91 \%$ da amostra com al to acúmulo de placa. Rivera-H ermosillo et al. ${ }^{17}$ também identificaram que os hábitos de saúde bucal dos adolescentes estudados deveriam ser modificados, devido à grandeingestão dealimentos cariogênicos e a baixa frequência de escovação. Isto demonstra que o problema com hábitos e condição de saúde bucal em adolescentes é geral e que estudos medindo a efetividade das ações são necessários. A divulgação de informações não garante a mudança de hábitos, por isso é importante avaliar as mudanças ocorridas nos hábitos e a condição de higiene bucal como ocorreu no estudo de Souza et al. ${ }^{19}$

N este estudo, observou-se melhora na higiene oral dos adolescentes que participaram das ações na escola, aumento no número de adolescentes que apresentaram gengiva saudável ou inflamação leve no segundo exame nos grupos escola e visita domiciliar. Com relação ao consumo dedoces, o grupo semanal e o da escola apresentaram diminuição entre o 10 e 20 exames.

Uma das limitações deste estudo foi o reduzido número deadolescentes pertencentes aos grupos $\mathrm{B}$ e $\mathrm{C}$ devido à dificuldade de acompanhamento controlado de um grande número de famílias pelos ACSs (relativo ao grupo $B$ ) e à dificuldade de se manter um número grande de pessoas participando regularmente das atividades em grupo (relativo ao grupo C). M esquini et al. ${ }^{20}$ ressaltaram a importância da abordagem de assuntos relacionados à saúde bucal no currículo escolar, proporcionando educação em saúde e ajudando a diminuir os índices de doenças como cárieegengivite.

N o planejamento das ações na escola tentouse utilizar o conceito ampliado de educação em saúde, levando em conta aintersetorialidade(uni- dade de saúde/escola), a interdisciplinaridade e a relação horizontal. 0 planejamento foi feito em conjunto com os professores e os temas foram trabalhados em todas as disciplinas. Os encontros foram realizados em forma de rodas de conversa, procurando trabalhar com o que fazia sentido para os adolescentes, sensibilizando-os da melhor forma em relação aos temas. Também foram utilizados recursos audiovisuais para facilitar o entendimento. Este conjunto de ações levou a um aumento do envolvimento dos adolescentes com as atividades possibilitando, maior efetividade no alcance de resultados.

Pauleto et al. ${ }^{21}$, ao reverem os principais programas educativos em saúde bucal identificaram que, apesar de muitos programas estarem surgindo nos últimos anos, a educação em saúde ainda é um desafio. A maioria dos programas priorizava tratamentos preventivos medicalizadores e curativos em detrimento da educação. Ainda revelaram que é preciso substituir modelos ancorados em práticas de comunicação unidirecional, dogmática e autoritária com foco na transmissão de informação, pela discussão e reflexão desencadeadas pela problematização. 0 estudo de Almeida e Ferreira ${ }^{22}$ confirma que essas práticas tradicionais ainda estão sendo eleitas pelos cirurgiões dentistas da ESF.

Koyashiki et al. ${ }^{23}$ apontam os Agentes Comunitários como profissionais que se utilizam de tecnologias leves (conversa, acolhimento, escuta solidaria e mediação), dando uma nova forma ao ato de cuidar. Percebe-se que o ACS é um elemento muito importante, contribuindo para o processo de transformação social. Entretanto, nesta pesquisa, observou-se que os melhores resultadosno queconcerneao aumento de frequência de escovação foram obtidos nas ações desenvolvidas na escola.

Algumas dificuldades foram encontradas peIos ACS, entre as quais se destacaram: impossibilidade de encontrar todos da família na hora da visita para orientação e falta de tempo para realizar as visitas já que exercem várias outras funções na Unidade de Saúde. U ma questão também importante é repensar as capacitações em saúde bucal para os agentes comunitários de saúdee o planejamento conjunto com estes profissionais para que as ações façam sentido e sejam desenvolvidas de forma eficaz.

No estudo de Oliveira e Albuquerque ${ }^{24}$, encontrou-se que os profissionais avaliam negativamente a ESF no tocante às condições de trabaIho fornecidas pelos gestores, a falta e/ ou insuficiência de capacitação e a sobrecarga de traba- 
Iho. A reflexão sobre a relação destes fatores na efetividade das ações faz-se necessária.

\section{Conclusões}

As ações educati vas realizadas na escola mostraram-se efetivas na mel hora das condições de saúde bucal e de hábitos, sendo importante o processo de capacitação e o planejamento conjunto das ações entre os profissionais da escola e os profissionais de saúde.

\section{Colaboradores}

\section{Referências}

APS Turrioni, FGD Salomão, JFC M onti, FL Vazquez, KL Cortellazzi eAC Pereira participaram igualmente de todas as etapas de elaboração do artigo.

1. Elias MS, Cano MAT, M estriner Júnior W, Ferriani M GC. A importância da saúde bucal para adolescentes de diferentes estratos sociais do município de Ribeirão Preto. Rev latino-am enfermagem 2001; 9(1):88-95.

2. Facó EF, Viana LMO, Bastos VA, Nuto SAS. O cirurgião dentista e o programa saúde da família na microrregião II, Ceará, Brasil. RBPS 2005; 18(2):70-77.

3. Pontes NST. Processo de trabalho em saúde bucal e necessidade de educação permanente: a experiência do PSF no município de Petrópolis [dissertação]. Rio de Janeiro (RJ): Universidade do Estado do Rio de Janeiro; 2007.

4. Rodrigues AAAO, Assis M M A. Oferta e Demanda na atenção à saúde bucal: o processo de trabalho no programa saúde da família em Alagoinhas - Bahia. Rev Baiana de Saúde Pública 2005; 29(2):273-285.

5. Araújo YP, Dimenstein M. Estrutura e organização do trabalho do cirurgião-dentista no PSF de municípios do Rio Grande do Norte. Cien Saude Colet 2006; 11(1):219-227.

6. Pace MA, Grigolleto JC, Bertoldi RC, Polachini M CGW, Bregagnolo JC. Hábitos de higiene oral de famílias cadastradas em programa de saúde da família de Ribeirão Preto - SP. Cad Saude Coletiva 2006; 14(1):49-62.

7. Brasil. M inistério da Saúde (MS). Cadernos de Atenção Básica: Saúde Bucal. № 17. Brasília: M S; 2006.

8. Flores EMTL, Drehmer TM. Conhecimentos, percepções, comportamentos e representações de saúde e doença bucal dos adolescentes de escolas públicas de dois bairros de Porto Alegre. Cien Saude Colet 2003; 8(3):743-752.

9. Tomita NE, Pernambuco RA, Lauris JRP, Lopes ES. Educação em Saúde Bucal para adolescentes: uso de métodos participativos. Rev. FOB 2001; 9(1/2):63-69. 
10. Ferrari RAP, Thonsom Z, M elchior R. Atenção à saúde dos adolescentes: percepção dos médicos e enfermeiros das equipes da saúde da família. Cad Saude Publica 2006; 22(11):2491-2495.

11. Organização Mundial da Saúde (OMS). In: Congresso M undial de Odontologia Preventiva 1993 set 35; U mea, Suécia; 1993.

12. Garcia RA, Matos R. A distribuição espacial da vulnerabilidade social das famílias brasileiras. In: Seminário População, Pobreza e Desigualdade; 2007 nov 5-7; Belo Horizonte (M G), Brasil; 2007.

13. Greene JC, Vermillion JR. The simplified oral hygiene index. J Am Dent Assoc 1964; 68:7-13.

14. Löe H, Silness J. Periodontal disease in pregnancy. I. prevalence and severity. Acta O dontol Scand 1963; 21(6):533-551.

15. Bardin L. Análise de conteúdo. Lisboa: Edições 70, 1979.

16. Santos NCN, Alves TDB, Freitas VS, Jamelli SR, Sarinho ESC. A saúde bucal de adolescentes: aspectos de higiene, de cárie dentária e doença periodontal nas cidades de Recife, Pernambuco e Feira de Santana, Bahia. Cien Saude Colet 2007; 12(5):1155-1166.

17. Rivera-Hermosillo G, M artínez-Torres J, Hernández-Laguna E. Caries dental e higiene bucal en adolescentes. M éxico Rev ADM 2006; 63(6):231-234.

18. Toassi RFC, Petry PC. Motivação no controle do biolfilme dental e sangramento gengival em escolares. Rev Saude Publica 2002; 36(5):634-637.

19. Souza GB, Nogueira de Sá PHR, Junqueira SR, Frias AC. Avaliação dos Procedimentos Coletivos em Saúde Bucal: percepção de adolescentes de Embu, SP. Rev Saúde Social 2007; 16(3):138-148.

20. Mesquini MA, Molinari SL, Prado IM M. Educação em saúde bucal: uma proposta para abordagem no Ensino Fundamental e M édio. Arq M udi. 2006; 10(3):16-22.

21. Pauleto ARC, Pereira M LT, Cyrino EG. Saúde bucal: uma revisão crítica sobre programações educativas para escolares. Cien Saude Colet 2004; 9(1): 121-130.

22. Almeida GCM, Ferreira MAF. Saúde bucal no contexto do Programa Saúde da Família: práticas de prevenção orientadas ao indivíduo e ao coletivo. Cad Saude Publica 2008; 24(9):2131-2140.

23. Koyashiki GAK, Alves-Souza RA, Garanhani ML. 0 trabalho em saúde bucal do Agente Comunitário de Saúde em Unidades de Saúde da Família. Cien Saude Colet 2008; 13(4):1343-1354.

24. Oliveira SF, Albuquerque, FJB. Programa de saúde da família: uma análise a partir das crenças dos seus prestadores de serviço. Psicologia \& Sociedade 2008; 20(2):237-246.

Artigo apresentado em 05/04/2011

Aprovado em 10/06/2011

Versão final apresentada em 26/07/2011 